\title{
Gemcitabine-loaded RGD modified liposome for ovarian cancer: preparation, characterization and pharmacodynamic studies
}

This article was published in the following Dove Press journal:

Drug Design, Development and Therapy

\section{Zhongyuan Tang \\ Weiwei Feng \\ Yiqing Yang \\ Qun Wang}

Department of Obstetrics \& Gynecology, Ruijin Hospital, Shanghai Jiaotong University School of Medicine, Shanghai, People's Republic of China
Correspondence: Weiwei Feng Department of Obstetrics \& Gynecology, Ruijin Hospital, Shanghai Jiaotong

University School of Medicine, No. 197,

Ruijin Er Road, Shanghai 200025, People's

Republic of China

$\mathrm{Tel}+862164370045$

Email fww12066@rjh.com.cn
Background: Ovarian cancer is the third leading cause of death among gynecological cancers in women in China. Chemotherapy is an important method for comprehensive treatment of ovarian cancer, but the curative effect is poor.

Purpose: In this study, gemcitabine (GEM) -loaded RGD modified liposomes (LPs) were developed by the emulsification-solvent evaporation method and evaluated for their antitumor activity in vitro and in vivo.

Methods: The physicochemical properties of LPs such as particle size, zeta potential and in vitro drug release were investigated. We also demonstrated the effect of RGD-GEM-PEG LPs in ovarian cancer.

Results: RGD-PEG3500-DSPE GEM LPs had a uniform spherical morphology. The mean particle size and polydispersity index were determined to be $106.7 \mathrm{~nm}$ and 0.13 respectively. The $\mathrm{ER} \%$ and $\mathrm{DL} \%$ of the formulation were $79.6 \pm 3.1 \%$ and $6.1 \pm 1.4 \%$ respectively. Compared with the free drug, RGD modified GEM LPs had sustained-release properties in vitro. In vivo, compared with the DiD-RGD-PEG3500-DSPE GEM LPs group, free DiD-GEM and DiDGEM LPs had no obvious fluorescence intensity in tumor of mice at all times, indicating that ordinary liposomes and drugs had no tumor targeting function. RGD-PEG3500-DSPE GEM LPs showed a superior antiproliferative effect on SKOV3 cells and had a better antitumor effect in vivo than non-modified LPs.

Conclusion: These results indicated that RGD-PEG3500-DSPE GEM LPs were a promising candidate for antitumor drug delivery.

Keywords: RGD, gemcitabine, liposomes, sustained-release, antitumor

\section{Introduction}

Ovarian cancer is the third leading cause of death among gynecological cancers in women in China. ${ }^{1,2}$ The lifetime risk of ovarian cancer is about $1.5 \%$, which is the second most common gynecologic malignancy. Because these cancers tend to be relatively aggressive and there are no proven early detection tests, most patients are not diagnosed until they have reached advanced stages (III or IV). ${ }^{3}$ This contributes to the poor prognosis observed in ovarian cancer, with a 5-year survival rate of only $35-38 \%$ at all stages. Standard treatment usually includes surgical staging and decompression, followed by chemotherapy. Chemotherapy is an important method for comprehensive treatment of ovarian cancer, ${ }^{4-6}$ but the curative effect is poor.

Gemcitabine (GEM) is one of the most important chemotherapeutic drugs for ovarian cancer, lung cancer and pancreatic cancer. ${ }^{7}$ It targets specific stages of cell cycle. There have been many studies on the clinical efficacy of GEM in treating ovarian cancer., ${ }^{8,9}$ 
However, due to its small molecular weight and high hydrophilicity, GEM has a short plasma half-life (17 min) and is rapidly decomposed into inactive products after administration. At the standard intravenous infusion dose of $1000 \mathrm{mg} / \mathrm{m}^{2}$, a patient's plasma GEM concentration drops to only $0.4 \mu \mathrm{g} /$ $\mathrm{mL} 1 \mathrm{~h}$ after administration, considerably below the $5 \mu \mathrm{g} / \mathrm{mL}$ optimal plasma concentration for cancer cell inhibition. ${ }^{10}$ Therefore, a larger dose is necessary to achieve effective plasma concentration but causes greater side effects.

In recent years, various drug delivery systems have been developed that can provide targeted delivery of anti-cancer drugs to the tumor tissue. Numerous types of formulations have been developed to deliver GEM at the target site, including PEGylated liposomes (LPs); thermosensitive liposomes; nanoparticles; solid lipid nanoparticles and albumin nanoparticles, et al. ${ }^{11-15}$ RGD is a short peptide containing arginine, glycine, and aspartic acid, which is expressed on the surface of various types of tumor, including ovarian, breast and pancreatic cancer. ${ }^{16-18}$ RGD peptides, as the recognition sites for integrins and their ligands, have adhesion between mediated cell, extracellular matrix and cells. Exogenous RGD peptides effectively inhibit the binding of ligands to integrins, thus inhibiting angiogenesis and migration of tumor cells. At the same time, tumor can be targeted and labeled, and anticancer drugs can be target delivered. ${ }^{19-21}$

According to the existing literature, there have been some reports on the study of GEM-loaded liposome and RGD modified GEM. ${ }^{22,23}$ However, there is no systematic report on the basic research of RGD modified GEM-loaded PEGylated liposome in ovarian cancer. In the present study, liposomes consisting of PEG were formed by the emulsification-solvent evaporation method. The physicochemical properties of RGD-GEM-PEG LPs such as particle size, zeta potential and in vitro drug release were investigated. We also demonstrated the effect of RGD-GEM-PEG LPs in ovarian cancer.

\section{Materials and methods}

\section{Materials}

GEM was gifted by Hengrui Pharmaceuticals Co Ltd (Jiangsu, China). RGD peptide (MW=1,100 Da) was purchased from Biochempartner (Shanghai, China). 1,2Distearoyl-sn-glycero-3-phosphoethanolamine (DSPE)$\mathrm{PEG}_{3500}-\mathrm{RGD}$ was synthesized following a previous report. $^{24}$ SKOV3 ovarian cancer cell lines were purchased from the Shanghai Institute of Biochemistry and Cell Biology. The chemical and solvents used were analytical or HPLC (Sinopharm Chemical Reagent Co., Ltd, China).
In this study, deionized water was used. Female Balb/c mice (5-6 weeks old, 25 \pm 2 g) were obtained from Laboratory Animal Center of Faculty of Pharmacy and Pharmaceutical Science, the Second Hospital of Anhui Medical University, China. All animals were kept in environment free of pathogens, with free access to food and water. Animal experiments were carried out in accordance with the guidelines issued by the National Institutes of Health and approved by the Second Hospital of Anhui Medical University.

\section{Preparation of RGD-PEG ${ }_{3500}$-DSPE GEM LPs}

RGD-PEG 3500 -DSPE GEM LPs were prepared by the high pressure homogenization method. Firstly, GEM (10 mg), phosphatidylcholine (125 mg), cholesterol (55 mg) and DSPE-PEG $_{3500}-$ RGD (2 mg) were dissolved in $12 \mathrm{~mL}$ chloroform-methanol $(3: 1, \mathrm{v} / \mathrm{v})$. Then chloroform-methanol was removed under $\mathrm{N}_{2}$ and evaporated in vacuum for at least $2 \mathrm{hrs}$ to form thin lipid membranes. A volume of $5 \mathrm{~mL}$ of phosphate buffered saline (PBS, $\mathrm{pH}$ 7.4) was added to the hydration lipids. The liposome suspensions were passed through a microfluidizer at 22,000 bar for ten circles, and then extruded (ten times) through polycarbonate membranes with gradually reduced pore size (200 and $100 \mathrm{~nm}$ ). Finally, RGD-PEG 3500 -DSPE GEM LPs were freeze-dried into powder. Non-modified preparations (GEM LPs) without (DSPE)-PEG $3500-$ RGD conjugate and corresponding blank LPs without GEM were prepared in a similar manner.

\section{Characterization}

RGD-PEG ${ }_{3500}$-DSPE GEM LPs lyophilized powders were redispersed separately in PBS $(\mathrm{pH}=7.4)$ and $\zeta$-potential was measured with a Malvern Zeta sizer 4 (Malvern Instruments, Worcestershire, UK). The size of the LPs was determined by photon correlation spectroscopy with a Malvern 4700 submicron particle analyzer system. TEM (H-600; Hitachi, Tokyo, Japan) was also used to study the morphology of LPs.

Lyophilized powder of RGD-PEG 3500 -DSPE GEM LPs was dispersed in an aqueous medium containing $0.5 \%(\mathrm{w} / \mathrm{v})$ Tween-80 to dissolve GEM. The GEM concentration in the supernatant after centrifugation was detected by HPLC. The drug encapsulation rate (ER\%) of LPs was equal to (total GEM-GEM in the supernatant)/total GEM $\times 100 \%$. 
Drug loading (DL\%) of LPs =

(weight of GEM in LPs / total weight of LPs) $\times 100 \%$

\section{In vitro drug release}

In this study, the dialysis bag (MWCO $=10,000)$ method was used to evaluate the in vitro release behavior of RGD-PEG ${ }_{3500^{-}}$ DSPE GEM LPs. Briefly, RGD-PEG 3500 -DSPE GEM LPs $(\approx 10 \mathrm{mg}$ GEM$)$ was sealed in a dialysis bag and immersed in $200 \mathrm{~mL}$ of PBS (pH 7.4) containing $10 \%(\mathrm{v} / \mathrm{v})$ fetal bovine serum (FBS) at $37{ }^{\circ} \mathrm{C}$ with a shaking rate of $100 \mathrm{r} / \mathrm{min}$. A sample $(1 \mathrm{~mL}$ each) was withdrawn from the medium at designated time intervals and the same volume of fresh medium was added. Each sample was centrifuged for $5 \mathrm{~min}$ at $12,000 \mathrm{rpm}$ and the supernatant was assayed by HPLC. The same doses of free GEM and GEM LPs were used as controls in this experiment.

\section{Evaluation of tumor targeting ability}

The in vivo tumor targeting ability of RGD-PEG 3500 -DSPE GEM LPs was evaluated by non-invasive optical imaging systems. Tumor-bearing mice models were built according to the previous report. Briefly, $1 \times 10^{9}$ SKOV3 cells were suspended in $200 \mu \mathrm{L}$ DMEM medium and injected into the right hindlimb flank of mice. DiD (NIRF dye, $50 \mathrm{nM}$ ) was co-loaded into RGD-PEG 3500 -DSPE GEM LPs as described above. When tumor volume reached $\sim 100-150 \mathrm{~mm}^{3}$, DiD-GEM, DiD-GEM LPs and DiD-RGD-PEG $3500^{-D S P E ~ G E M ~ L P s ~ i n ~}$ $100 \mu \mathrm{L}$ PBS were injected intravenously via the tail vein in tumor bearing mice. At different time points (12 and $24 \mathrm{~h}$ ), mice were anesthetized by intraperitoneal injection of pentobarbitol $(35 \mathrm{mg} / \mathrm{kg})$, and scanned using the imaging system IS4000MM (Kodak) with an excitation bandpass filter at $625 \mathrm{~nm}$ and an emission at $700 \mathrm{~nm}$. Exposure time was $30 \mathrm{~s}$ per image.

\section{Cell uptake}

The SKOV3 cell lines were seeded into 24-well plates at a density of $1 \times 10^{6}$ cells $/ \mathrm{mL}$. After $24 \mathrm{~h}$, each well was subsequently incubated with $1 \mathrm{~mL}$ of a $100 \mathrm{mg} / \mathrm{mL}$ coumarin-6loaded free GEM, GEM-LPs and RGD-PEG 3500 -DSPE GEM LPs for $2 \mathrm{~h}$. For quantitative analysis, the suspension was removed at the designated time period, and the wells were washed three times with a $1000 \mu \mathrm{l}$ cold PBS. Following this, $50 \mu 1$ of $0.5 \%$ Triton X-100 was added into each well for cell lysis. The fluorescence intensity of each sample well was measured by a microplate reader. The excitation wavelength was $465 \mathrm{~nm}$ and emission wavelength was $502 \mathrm{~nm}$. In the qualitative study, the cells were washed with cold PBS three times and immobilized with 4\% paraformaldehyde for 20 mins. Then, the cells were washed with cold PBS twice and observed by confocal laser scanning microscopy.

\section{MTT assay for cell viability}

Briefly, SKOV3 cells $\left(5 \times 10^{3}\right.$ per well) were seeded in 96 -well plates. After being subcultured for $24 \mathrm{~h}$ and $48 \mathrm{~h}$, the cells were treated with free GEM of different concentrations $(0.2,0.57$, 0.95, $2 \mathrm{mmol} / \mathrm{L}$ ) or GEM LPs, RGD-PEG 3500 -DSPE GEM LPs with GEM of equivalent concentrations. Blank RGD$\mathrm{PEG}_{3500}$-DSPE LPs were used as the control group. Cells were incubated for $48 / 72 \mathrm{~h}$ and cell viability was assessed by MTT (3-(4,5-dimethyl-thiazol-2-yl)-2,5-diphenyltetrazolium bromide) assay as described below. $1 \mathrm{~mL}$ complete growth culture medium and $50 \mu \mathrm{l}$ MTT solution $(2 \mathrm{mg} / \mathrm{mL}$ in PBS) were added to each well and incubated for 4 hrs. Finally, the absorbance of each well was measured at $570 \mathrm{~nm}$. The results were expressed as a percentage relative to the result obtained with a non-toxic control. Experiments were carried out in triplicate.

\section{In vivo evaluation}

The anticancer effect of RGD-PEG 3500 -DSPE GEM LPs was demonstrated in an ovarian cancer xenografts model established as previously described. ${ }^{23} 24$ ovarian cancer-bearing mice were randomly divided into the following four treatment groups (6 mice per group): PBS (control), free GEM, GEM LPs and RGD-PEG 3500 -DSPE GEM LPs. For each treatment group, an equivalent dose of $100 \mu \mathrm{g} / \mathrm{kg}$ GEM was injected into the mice's caudal veins on day 12, 15, 18, 21, 24, 27 and 30 after tumor inoculation. After the initial administration, the tumor diameter was measured by a dial caliper every three days. The subcutaneous tumor volume was estimated by the following formula: $\mathrm{V}\left(\mathrm{mm}^{3}\right)=\left(\right.$ length $\times$ width $\left.^{2}\right) / 2$. All mice were euthanized and the tumor tissues were harvested for further protein analysis until the 42th day after tumor inoculation. The bcl-2, bax and caspase 3 protein expression of the tumor were characterized by Western blot analysis. The analysis results were expressed in the format of mean \pm standard deviation (SD) as the ratio percentage of the protein of interest of optical density versus the $\beta$-actin optical density.

\section{Pharmacokinetic study}

All the in vivo experimental protocols were approved by the animal care committee of Shanghai Jiaotong University School of Medicine and all experiments were conducted in strict accordance with the laboratory animal care and usage 
guidelines adopted by the national institutes of health (Shanghai, China). The pharmacokinetics of GEM preparations was observed in 18 rats after intravenous administration. Rats were randomly divided into 3 groups, given a $10 \mathrm{mg} / \mathrm{kg}$ intravenous dose of GEM injection, GEM LPs and RGD$\mathrm{PEG}_{3500}$-DSPE GEM LPs via the tail vein. Blood samples $(0.5 \mathrm{~mL})$ were collected from the orbital cavity into heparinized tubes at $0.25,0.5,1,2,4,6,8,10,12$ and $24 \mathrm{~h}$ after i.v. administration. The blood was immediately processed for plasma by centrifugation at $2000 \times \mathrm{g}$ for $10 \mathrm{~min}$. Plasma samples were frozen at $-70{ }^{\circ} \mathrm{C}$ until analysis.

\section{Statistical analysis}

The results were analyzed using ANOVA. Significant differences (if $p<0.05$ ) between groups were identified using Student-Newman multiple comparison post hoc test (GraphPad Instat software - GraphPad).

\section{Result and discussion}

\section{Preparation and characterization}

In this study, RGD-PEG 3500 -DSPE GEM LPs were prepared by the high pressure homogenization method and a number of factors were investigated to optimize the formulation parameter. The solid lipid, modified material and drugs were mixed and dispersed in the organic phase under the condition of hot melt, and then LPs with a certain size distribution were prepared by homogenizing the high pressure homogenizer (Figure 1A). Compared with other methods, the high pressure homogenization method has advantages such as simple operation, easy control of process parameters and suitability for industrial production. The process of parameter selection indicated that the amount of organic phase (chloroform-methanol) significantly influenced the preparation of LPs. When the ratio of organic phase to solid lipid (phosphatidylcholine+cholesterol) was greater than or equal to $1: 15$ (mL:mg), the yield of LPs was no less than $82 \%$; however, as the ratio of organic phase to solid lipid increased, the mean diameter of LPs grew significantly.

As shown in Figure 1B, RGD-PEG 3500 -DSPE GEM LPs had a uniform spherical morphology. The mean particle size and polydispersity index were $106.7 \mathrm{~nm}$ and 0.13 respectively. According to literature, when the particle size of LPs was less than $400 \mathrm{~nm}$, the LPs could exudate into the tumor microspace, and smaller sizes could be more effectively internalized through endocytic vesicles. ${ }^{25}$ In this study, the $\zeta$-potential of RGD-PEG 3500 -DSPE GEM LPs was $-24.6 \mathrm{mV}$, which might be resulted from the structure of the ionic double layer and produced a higher zeta potential value. The ER\% and DL\% of the formulation were $79.6 \pm 3.1 \%$ and $6.1 \pm 1.4 \%$ respectively.

\section{In vitro drug release}

The in vitro drug release curve of RGD-PEG 3500 -DSPE GEM LPs was shown in Figure 2. The release of free GEM and GEM LPs was also investigated as a control. In vitro release data showed that free GEM was released faster than ordinary GEM LPs and RGD-PEG 3500 -DSPE GEM LPs, and almost $90 \%$ of free GEM was released after 2 hrs. There was an obvious distinct burst release phenomenon in the release of both GEM LPs. Burst release lasted 30 mins after administration; then the release gradually slowed down. The rates of burst release were $13.25 \% \pm 3.05 \%$ and $17.13 \% \pm 2.29 \%$ for RGD-PEG $3500^{-}$ DSPE GEM LPs and GEM LPs respectively. It could be concluded that the release rate of the drug was largely a function of its localization within the LPs. The outer corona region of the LPs was quite mobile. As a result, release from this area should be rapid. The release of drug localized in the corona, or at the interface, was believed to account for "burst release" from the LPs. Moreover, no significant changes were found in terms of release characteristics when RGD-PEG 3500 -DSPE was added to the LPs.
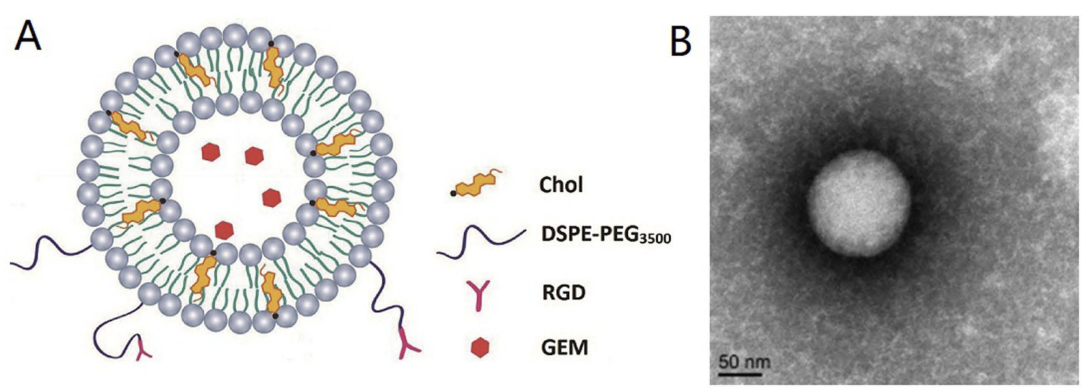

Figure I The structure of RGD-PEG 3500 -DSPE GEM LPs $(\mathbf{A})$ and the transmission electron microscope of RGD-PEG 3500 -DSPE GEM LPs $(\mathbf{B})(\times 50,000)$. 


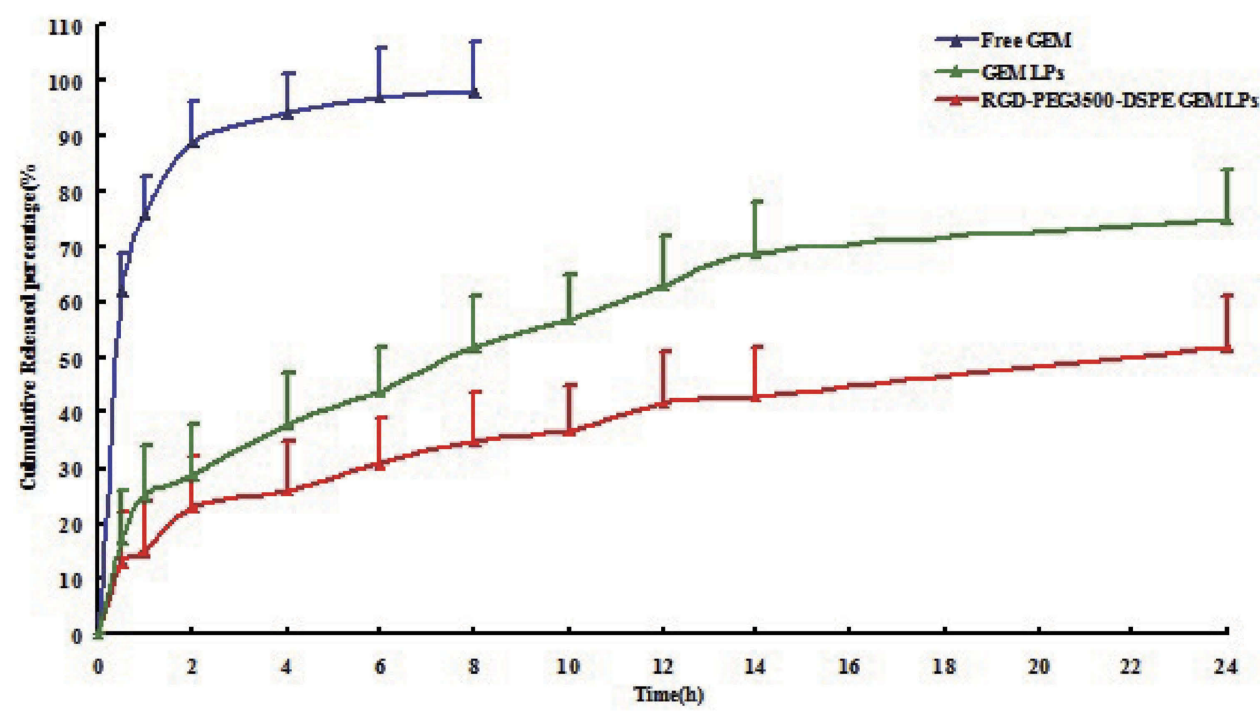

Figure 2 The release profile of free GEM, GEM LPs, RGD-PEG 3500 -DSPE GEM LPs $(n=6)$.

\section{Evaluation of tumor targeting ability}

The tumor targeting efficiency of DiD fluorescence labeled RGD-PEG 3500 -DSPE GEM LPs in mice bearing s.c. SKOV3 ovarian cancer xenograft was monitored by non-invasive fluorescence optical imaging. Equivalent amounts of DiD-GEM, DiD-GEM LPs and DiD-RGD-PEG ${ }_{3500}$-DSPE GEM LPs were injected into mice through tail vein. The entire animal became fluorescent immediately after nanoparticle administration, and substantial contrast between subcutaneous tumor and normal tissue was observed from 0 to $24 \mathrm{~h}$ (Figure 3). According to the imaging results, the uptake of DiD-RGD$\mathrm{PEG}_{3500}$-DSPE GEM LPs in tumors increased gradually compared to normal tissue following injection, peaking at $12 \mathrm{hrs}$ and maintaining a strong fluorescence signal for $24 \mathrm{hrs}$. This suggested that DiD-RGD-PEG 3500 -DSPE GEM LPs were more likely to accumulate in tumors than normal tissues, which may be due to prolonged circulation and enhanced permeability and retention (EPR) effects. Compared with the
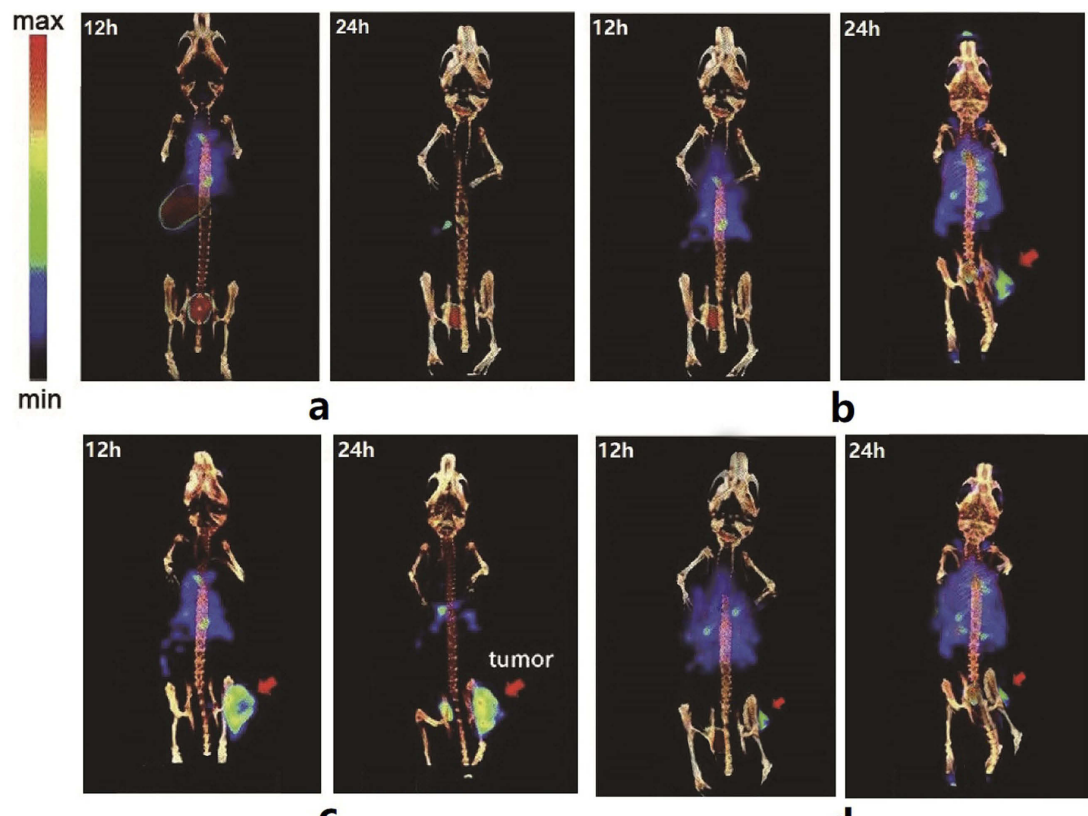

Figure 3 Fluorescence images of subcutaneous SKOV3 tumor-bearing nude mice after intravenous injection of free GEM (A), GEM LPs (B), RGD-PEG 3500 -DSPE GEM LPs (C) and free RGD peptide intravenously inject prior to DiD-RGD-PEG 3500 -DSPE GEM LPs injection (D). $12 \mathrm{~h}$ and $24 \mathrm{~h}$ after injection. 
DiD-RGD-PEG 3500 -DSPE GEM LPs group, free DiD-GEM and DiD-GEM LPs had no obvious fluorescence intensity in tumor of mice at any time, indicating that ordinary LPs and drugs had no tumor targeting function. Meanwhile, it can be found that if free RGD peptide intravenously inject prior to DiD-RGD-PEG ${ }_{3500}$-DSPE GEM LPs injection, the drug concentration of GEM is obviously lower at two different time points.

\section{Cell uptake}

The SKOV3 cell lines had the ability to take up the coumarin-6-loaded free GEM, GEM-LPs and RGD-PEG ${ }_{3500^{-}}$ DSPE GEM LPs at various capacities (Figure 4A-C). In SKOV3 cells, the fluorescence intensity of free GEM was the lowest among the three formulations. The fluorescence intensity of GEM-LPs was a little stronger than that of free GEM. However, RGD-PEG 3500 -DSPE GEM LPs uptake was markedly higher than that of GEM-LPs (approximately 2.5 folds higher), which was proposed to be the result of the targeting capacity of $\alpha v \beta 3$ integrin. Quantitative analysis indicated results very similar to those obtained from fluorescence imaging (Figure 4D).

\section{MTT assay for cell viability}

To evaluate the antiproliferative effects from various GEM LPs formulations, an MTT assay was performed in SKOV3 cells over 48 hrs of treatment (Figure 5). The viability of SKOV3 cells decreased with the increase of incubation time as well as GEM concentration. LPs exhibited higher toxicity following conjugation with RGD, as $\alpha v \beta 3$ integrin was overexpressed in SKOV3 cells. $^{26}$ From cell viability experiments, free GEM, GEM LPs and RGD-PEG 3500 -DSPE GEM LPs showed a time- and dose-dependent cytotoxic activity on SKOV3 cells. It is noteworthy that the RGD-PEG $3500^{-}$ DSPE GEM LPs formulation achieved the lowest cell viability in all three preparations at all equivalent drug concentration levels. This further confirmed the advantages for cellular uptake shown in the previous experiments, which resulted from the activation of the $\alpha v \beta 3$ integrin, thus contributing to an additional pathway through which the drug could be delivered into the cell cytoplasm to induce cell apoptosis.

\section{In vivo evaluation}

A nude mouse model of ovarian cancer was successfully established by subcutaneous injection of SKOV3 $\left(0.5 \times 10^{9}\right)$ cells. After inoculation, the mice at the early stage were in good condition, but their activities and eating began to decrease 2 weeks after inoculation, especially in the control group. At the end of the experiment, all animals died of tumor progression without apparent toxicity. The tumor nodules were
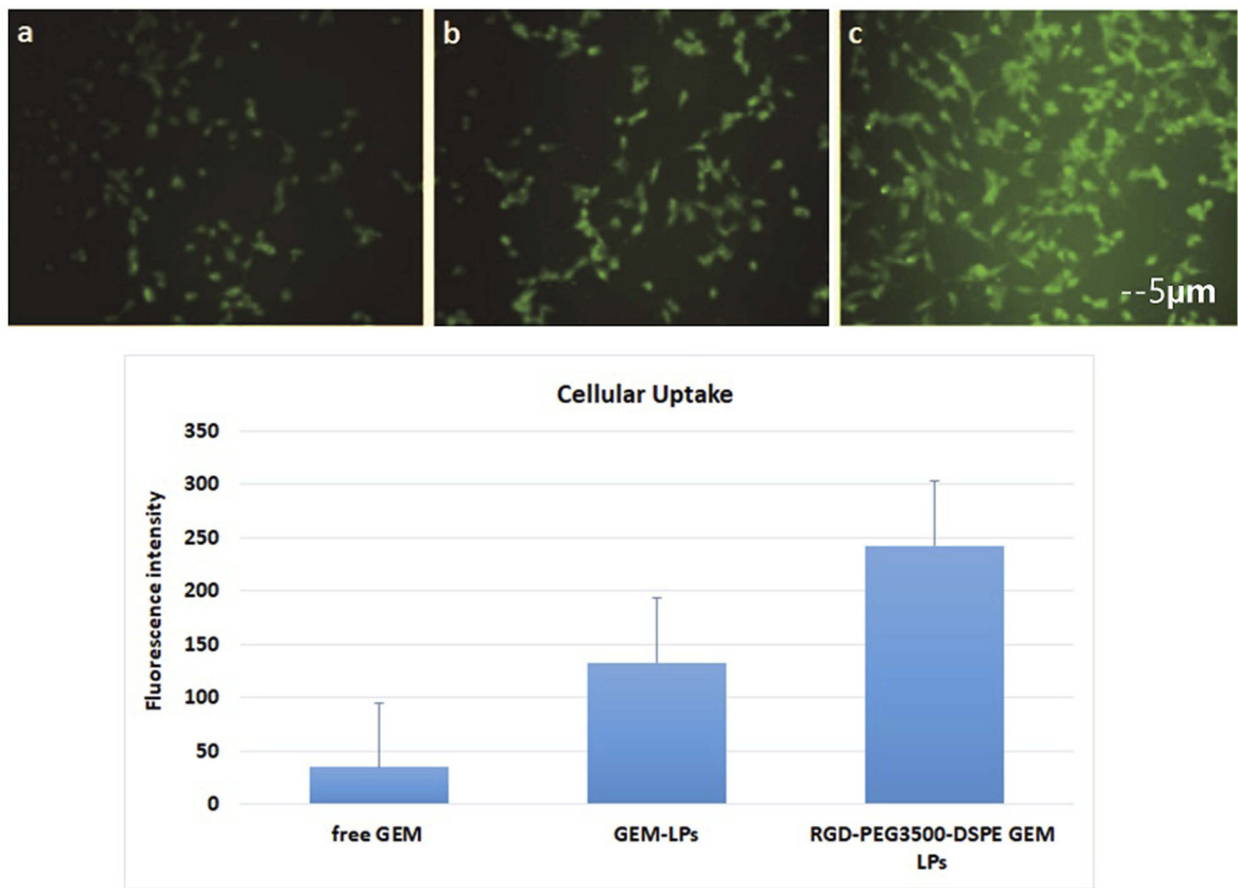

Figure 4 Confocal images of cellular uptake of free GEM (a), GEM LPs (b), RGD-PEG $3500-$ DSPE GEM LPs (c) by SKOV3 cells. Incubation time was 2 hrs. 

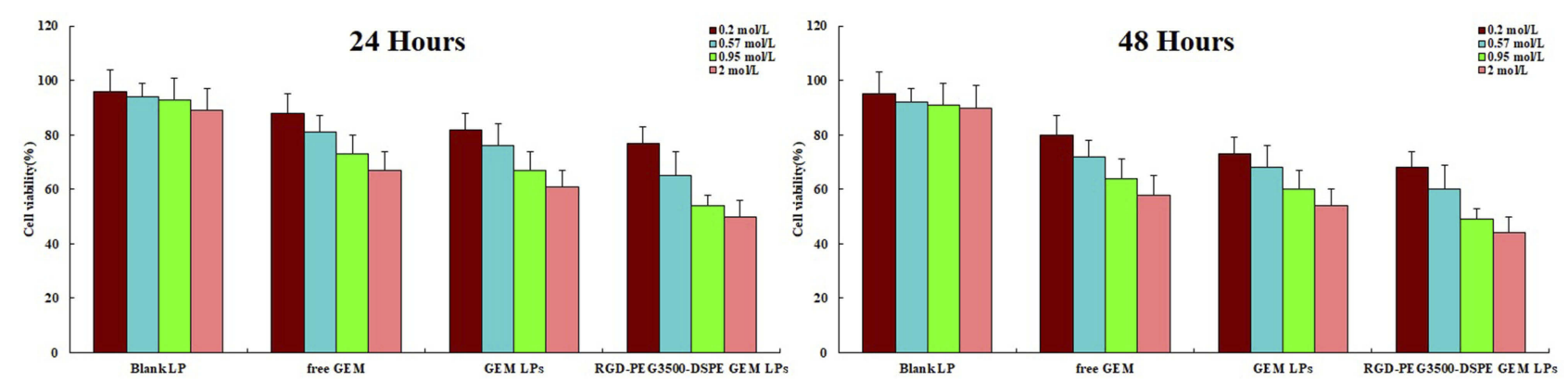

Figure 5 In vitro cytotoxicity analysis of free GEM, GEM LPs, RGD-PEG 3500 -DSPE GEM LPs on SKOV3 cell lines. Cell viability assay was performed by MTT assay.

palpable 7 days and visible 12 days after inoculation. The RGD-PEG 3500 -DSPE GEM LPs group exhibited the lowest level of tumor volume, and this was further verified by calculating the relative tumor volumes in different treatment groups at different time point (Figure 6A). The volume of tumor in the experimental group was significantly smaller than that in the PBS control group $(p<0.05)$, and the tumor volume of the mice in the RGD-PEG ${ }_{3500}$-DSPE GEM LPs group grew at the lowest speed $(p<0.05)$. Western blot was used to detect the expression of apoptosis related proteins Bcl-2, Bax and Caspase 3. Figure 6B and $\mathrm{C}$ showed the expression levels of Bcl-2, Bax and Caspase 3 in different treatment groups. The indexes represented bcl-2/ $\beta$-action; bax $/ \beta$-action and caspase $3 / \beta$-action respectively. Compared with the other treatment groups, RGD-PEG ${ }_{3500}$-DSPE GEM LPs group had the highest expression of bax and caspase- 3 and the lowest expression of

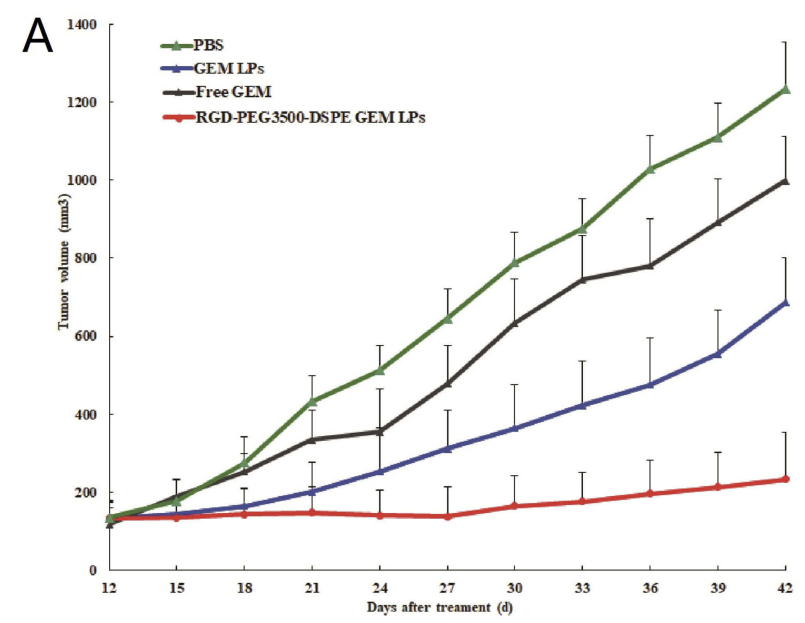

B

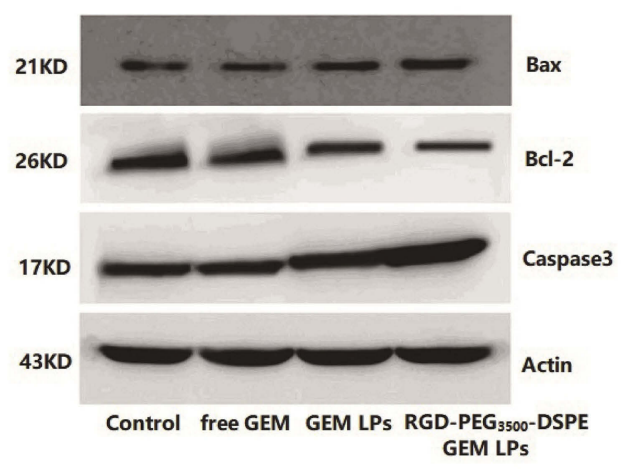

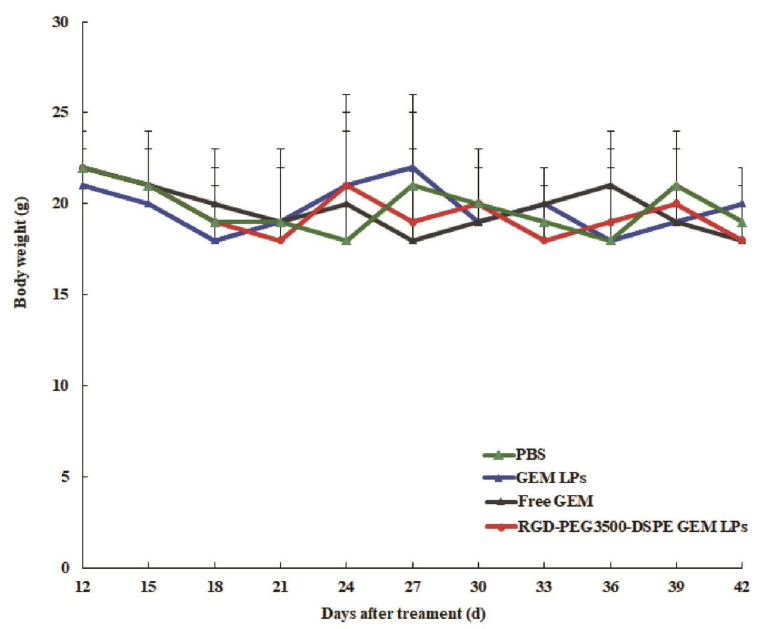

C

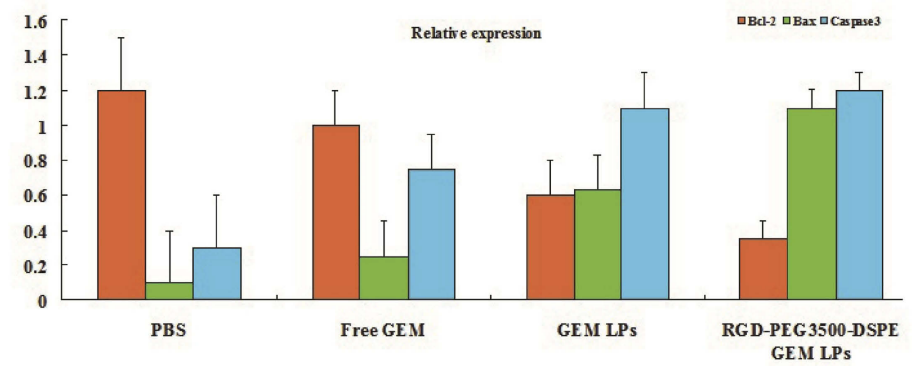

Figure 6 (A) SKOV3 xenograft tumor growth inhibition by GEM in different formulations; (B) Western blot analysis of the expression bcl-2, bax and caspase 3 protein in tumor tissue after different treatments; (C) The expression levels of bcl-2, bax and caspase 3 protein in different treatments groups. 
bcl-2, and the differences were statistically significant $(p<0.05)$. These results showed that RGD-PEG $3500-\mathrm{DSPE}$ GEM LPs significantly upregulated pro-apoptotic protein bax and downregulated anti-apoptotic protein bcl-2 expression, and increased apoptosis related protein caspase 3 expression. There are several possible reasons for the above results: 1) RGD acts as anchorage to specifically locate LPs in tumor sites and promote receptor-mediated endocytosis. 2) the $\mathrm{PEG}_{3500^{-}}$ DSPE anchored to the surface of GEM-LPs can avoid the rapid uptake with the reticuloendothelial system, thereby prolonging the circulation time and leading to a higher accumulation of LPs in the tumor vascular system. 3) the optimal particle size of the RGD-PEG 3500 -DSPE GEM LPs plays an important role in enhancing the permeability and retention effect.

Chemotherapeutics loaded in LPs thus far include doxorubicin, camptothecin, and fluorouracil. This is the first published study on GEM-loaded RGD modified

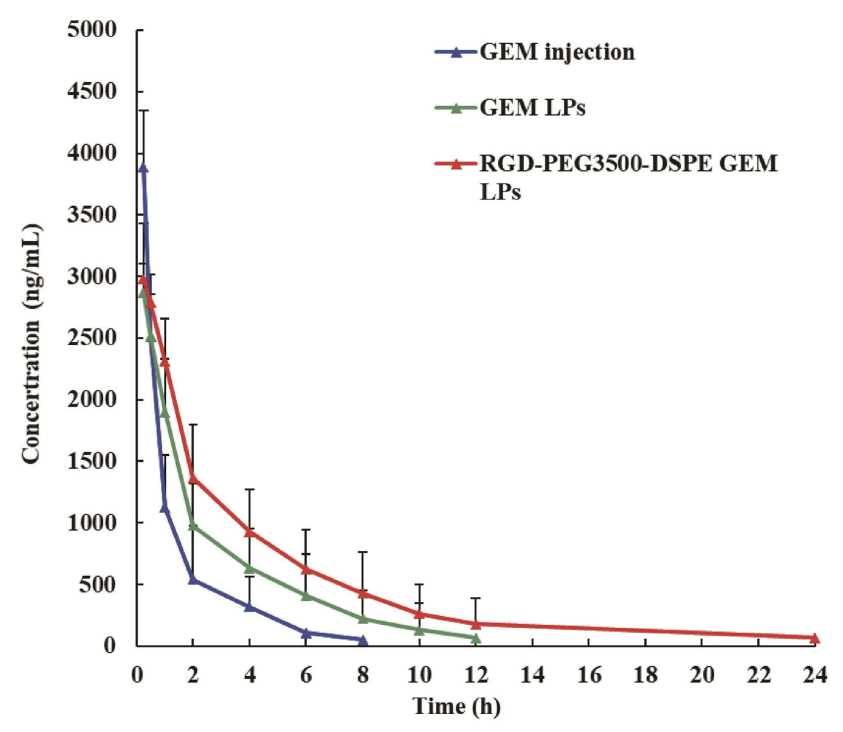

Figure 7 The time course of the plasma concentrations of GEM injection, GEM LPs and RGD-PEG3500-DSPE GEM LPs.
LPs. In this study, RGD-PEG3500-DSPE GEM LPs have the potential to be used to treat ovarian cancer. Further improvements are necessary for the RGDPEG3500-DSPE GEM LPs system, such as pancreatic and non-small-cell lung cancers. These challenges are the subject of our ongoing experiments.

\section{Pharmacokinetic studies}

Pharmacokinetic studies were carried out in rats using different GEM formulations. The time course of the plasma concentrations of GEM injection, GEM LPs and RGD-PEG 3500 -DSPE GEM LPs were summarized in Figure 7. Table 1 listed the pharmacokinetic parameters calculated from the plasma drug concentration vs time profiles.

There were significant differences in $t_{1 / 2}, \mathrm{AUC}_{0-\mathrm{t}}$, $\mathrm{AUC}_{0-\infty}$, MRT and CL between the GEM injection and RGD-PEG ${ }_{3500}$-DSPE GEM LPs $(p<0.05)$. As shown, after the single GEM injection, the plasma drug concentration quickly reached the maximum level $(3892.3 \pm 325.6 \mathrm{ng} / \mathrm{mL})$ in $15 \mathrm{~min}$, before it decreased rapidly, leaving around $13 \%$ of the Cmax value $2 \mathrm{~h}$ later. This implied that a rapid in vivo elimination of GEM existed in rats. In the case of intravenous administration, the in vivo profile of RGD-PEG ${ }_{3500^{-}}$ DSPE GEM LPs was smoother than the GEM injection group. The $t_{1 / 2}$ and $\mathrm{AUC}_{0-\infty}$ of RGD-PEG ${ }_{3500}-\mathrm{DSPE}$ GEM LPs were 3.13 and 2.51 folds high respectively compared with free drug. Therefore, it was reasonable to conclude that LPs could significantly extend the role of GEM in vivo (provided higher bioavailability). Meanwhile, RGD$\mathrm{PEG}_{3500}$-DSPE GEM LPs provided higher AUC ${ }_{0-\infty}$, MRT, and $t_{1 / 2}$ when compared with GEM LPs. However, RGD$\mathrm{PEG}_{3500}$-DSPE GEM LPs showed decreased clearance when compared with the GEM LPs. There was no significant difference in pharmacokinetic parameters between the two liposomes.

Table I Pharmacokinetic parameters of GEM after intravenous administration of GEM injection, GEM LPs and RGD-PEG $3500-\mathrm{DSPE}$ GEM LPs to rats. $(n=6)$

\begin{tabular}{|c|c|c|c|}
\hline \multirow[t]{2}{*}{ Parameter } & \multicolumn{3}{|c|}{ Intravenous administration } \\
\hline & GEM injection & GEM LPs & RGD-PEG $_{3500}$-DSPE GEM LPs \\
\hline$t_{1 / 2}(h)$ & $0.52 \pm 0.12$ & $1.23 \pm 0.65$ & $1.63 \pm 1.11^{\mathrm{a}}$ \\
\hline$A \cup C_{0-t}(n g \cdot h / m L)$ & $4540.5 \pm 418.7$ & $7428.8 \pm 825.7$ & $10,250.5 \pm \mid 121.4^{a}$ \\
\hline$A \cup C_{0-\infty}(\mathrm{ng} \cdot \mathrm{h} / \mathrm{mL})$ & $4827.9 \pm 538.6$ & $8172.6 \pm 912.2$ & $12,102.3 \pm 1213.6^{\mathrm{a}}$ \\
\hline MRT (h) & $0.53 \pm 0.21$ & $1.02 \pm 0.54$ & $1.33 \pm 1.01^{\mathrm{a}}$ \\
\hline$C L(\mathrm{~L} / \mathrm{kg} / \mathrm{h})$ & $0.4 I \pm 0.13$ & $0.17 \pm 0.07$ & $0.07 \pm 0.0 \mathrm{I}^{\mathrm{a}}$ \\
\hline
\end{tabular}

Notes: ${ }^{a} p<0.05$ : VS GEM injection; $t_{1 / 2}$, half-life; $A \cup C_{0-t}$, Area under the concentration-time curve from time 0 to the final measurable concentration.

Abbreviations: $\mathrm{AUC}_{0}$, area under the concentration-time curve pushed to infinity; MRT, mean residence time; $\mathrm{CL}$, Plasma clearance. 


\section{Conclusion}

To sum up, GEM LPs modified by RGD have a uniform spherical shape, and appropriate particle size and zeta potential. Compared with non-targeted LPs and free GEM, RGD$\mathrm{PEG}_{3500}$-DSPE GEM LPs can significantly enhance specific intracellular uptake and the apoptosis of SKOV3 cells in vitro. Meanwhile, RGD-PEG 3500 -DSPE GEM LPs have a significantly stronger ability of inhibiting tumor growth and inducing tumor apoptosis in vivo.

\section{Acknowledgment}

This study received funding from Shanghai Municipal Education Commission-Gaofeng Clinical Medicine Grant Support (20172003) to W. Feng.

\section{Disclosure}

The authors report no conflicts of interest in this work.

\section{References}

1. Peng Y, Lin J, Guan J, et al. Ovarian collision tumors: imaging findings, pathological characteristics, diagnosis, and differential diagnosis. Abdom Radiol (NY). 2018;43(8):2156-2168.

2. Gong W, Luo S, Hu R, et al. Analysis of survival rate of breast, cervical, and ovarian cancer patients during 2005-2010 in Zhejiang province, China. Zhonghua Yu Fang Yi Xue Za Zhi. 2014;48(5):366-369.

3. Goff BA, Mandel L, Muntz HG, Melancon CH. Ovarian carcinoma diagnosis. Cancer. 2000;89(10):2068-2075. doi:10.1002/1097-0142 (20001115)89:10<2068::aid-cncr6>3.0.co;2-z

4. Polom K, Roviello G, Generali D, et al. Cytoreductive surgery and hyperthermic intraperitoneal chemotherapy for treatment of ovarian cancer. Int $J$ Hyperthermia. 2016;32(3):298-310. doi:10.3109/ 02656736.2016 .1149233

5. Bukowska B, Rogalska A, Marczak A. New potential chemotherapy for ovarian cancer - combined therapy with WP 631 and epothilone B. Life Sci. 2016;151:86-92. doi:10.1016/j.1fs.2016.02.095

6. Cowan RA, O'Cearbhaill RE, Zivanovic O, Chi DS. Current status and future prospects of hyperthermic intraoperative intraperitoneal chemotherapy (HIPEC) clinical trials in ovarian cancer. Int J Hyperthermia. 2017;33 (5):548-553. doi:10.1080/02656736.2017.1283066

7. Mini E, Nobili S, Caciagli B, Landini I, Mazzei T. Cellular pharmacology of gemcitabine. Ann Oncol. 2006;17:v7-v12. doi:10.1093/ annonc/mdj941

8. Tomita Y, Saito T, Okadome M, Eto T, Ariyoshi K, Shimamoto K. The safety and efficacy of cisplatin plus gemcitabine in recurrent ovarian cancer. Int J Clin Oncol. 2014;19(4):662-666. doi:10.1007/s10147013-0599-5

9. Dijkgraaf EM, Santegoets SJ, Reyners AK, et al. A phase 1/2 study combining gemcitabine, pegintron and $\mathrm{p} 53$ SLP vaccine in patients with platinum-resistant ovarian cancer. Oncotarget. 2015;6 (31):32228-32243. doi:10.18632/oncotarget.4772
10. Lilly E. Co: Summary of Product Characteristics: Gemcitabine UK Prescribing Information. Indianapolis: Eli Lilly; 1997.

11. Xu H, Paxton J, Lim J, et al. Development of high-content gemcitabine PEGylated liposomes and their cytotoxicity on drug-resistant pancreatic tumour cells. Pharm Res. 2014;31(10):2583-2592. doi:10.1007/s11095-014-1353-z

12. May JP, Ernsting MJ, Undzys E, Li SD. Thermosensitive liposomes for the delivery of gemcitabine and oxaliplatin to tumors. Mol Pharm. 2013;10(12):4499-4508. doi:10.1021/mp400321e

13. Khaira R, Sharma J, Saini V. Development and characterization of nanoparticles for the delivery of gemcitabine hydrochloride. ScientificWorldJournal. 2014;2014:560962. doi:10.1155/2014/ 560962

14. Nandini PT, Doijad RC, Shivakumar HN, Dandagi PM. Formulation and evaluation of gemcitabine-loaded solid lipid nanoparticles. Drug Deliv. 2015;22(5):647-651. doi:10.3109/ 10717544.2013.860502

15. Dubey RD, Alam N, Saneja A, et al. Development and evaluation of folate functionalized albumin nanoparticles for targeted delivery of gemcitabine. Int $J$ Pharm. 2015;492(1-2):80-91. doi:10.1016/j. ijpharm.2015.07.012

16. Ji S, Xu J, Zhang B, et al. RGD-conjugated albumin nanoparticles as a novel delivery vehicle in pancreatic cancer therapy. Cancer Biol Ther. 2012;13(4):206-215. doi:10.4161/cbt.13.4.18692

17. Gamble LJ, Borovjagin AV, Matthews QL. Role of RGD-containing ligands in targeting cellular integrins: applications for ovarian cancer virotherapy (Review). Exp Ther Med. 2010;1(2):233-240. doi:10.3892/etm_00000037

18. Chakravarty R, Chakraborty S, Dash A. Molecular imaging of breast cancer: role of RGD peptides. Mini Rev Med Chem. 2015;15 (13):1073-1094.

19. Danhier F, Le Breton A, Préat V. RGD-based strategies to target alpha(v) beta(3) integrin in cancer therapy and diagnosis. Mol Pharm. 2012;9:2961-2973. doi:10.1021/mp3002733

20. Garanger E, Boturyn D, Dumy P. Tumor targeting with RGD peptide ligands - design of new molecular conjugates for imaging and therapy of cancers. Anticancer Agents Med Chem. 2007;7:552-558. doi:10.2174/187152007781668706

21. Zitzmann S, Ehemann V, Schwab M. Arginine-glycine-aspartic acid (RGD)-peptide binds to both tumor and tumor-endothelial cells in vivo. Cancer Res. 2002;62:5139-5143.

22. Han H, Valdepérez D, Jin Q, et al. Dual enzymatic reaction-assisted gemcitabine delivery systems for programmed pancreatic cancer therapy. ACS Nano. 2017;11(2):1281-1291. doi:10.1021/acsnano.6b05541

23. Matsumoto K, Onda T, Yaegashi N. Pharmacotherapy for recurrent ovarian cancer: current status and future perspectives. Jpn J Clin Oncol. 2015;45(5):408-410. doi:10.1093/jjco/hyv014

24. Mei L, Fu L, Shi K, et al. Increased tumor targeted delivery using a multistage liposome system functionalized with RGD, TAT and cleavable PEG. Int J Pharm. 2014;468(1-2):26-38. doi:10.1016/j. ijpharm.2014.04.008

25. Sahay G, Alakhova DY, Kabanov AV. Endocytosis of nanomedicines. $J$ Control Release. 2010;145(3):182-195. doi:10.1016/j.jconrel.2010.04. 020

26. Yang G, Sun H, Kong Y, Hou G, Han J. Diversity of RGD radiotracers in monitoring antiangiogenesis of flavopiridol and paclitaxel in ovarian cancer xenograft-bearing mice. Nucl Med Biol. 2014;41 (10):856-862. doi:10.1016/j.nucmedbio.2014.08.008 


\section{Publish your work in this journal}

Drug Design, Development and Therapy is an international, peerreviewed open-access journal that spans the spectrum of drug design and development through to clinical applications. Clinical outcomes, patient safety, and programs for the development and effective, safe, and sustained use of medicines are a feature of the journal, which has also been accepted for indexing on PubMed Central. The manuscript management system is completely online and includes a very quick and fair peer-review system, which is all easy to use. Visit http://www. dovepress.com/testimonials.php to read real quotes from published authors. 